\title{
Notes About the Carathéodory Number
}

\author{
Imre Bárány • Roman Karasev
}

Received: 28 December 2011 / Revised: 18 June 2012 / Accepted: 2 July 2012 /

Published online: 20 July 2012

(C) Springer Science+Business Media, LLC 2012

\begin{abstract}
In this paper we give sufficient conditions for a compactum in $\mathbb{R}^{n}$ to have Carathéodory number less than $n+1$, generalizing an old result of Fenchel. Then we prove the corresponding versions of the colorful Carathéodory theorem and give a Tverberg-type theorem for families of convex compacta.
\end{abstract}

Keywords Carathéodory's theorem · Helly's theorem · Tverberg's theorem

\section{Introduction}

The Carathéodory theorem [7] (see also [10]) asserts that every point $x$ in the convex hull of a set $X \subset \mathbb{R}^{n}$ is in the convex hull of one of its subsets of cardinality at most $n+1$. In this note we give sufficient conditions for the Carathéodory number to be less than $n+1$ and prove some related results. In order to simplify the reasoning, we always consider compact subsets of $\mathbb{R}^{n}$.

\section{Bárány}

Rényi Institute of Mathematics, Hungarian Academy of Sciences, P.O. Box 127, 1364 Budapest, Hungary

e-mail: barany@ renyi.hu

\section{Bárány}

Department of Mathematics, University College London, Gower Street, London WC1E 6BT, England

R. Karasev ( $₫)$

Dept. of Mathematics, Moscow Institute of Physics and Technology, Institutskiy per. 9, Dolgoprudny 141700, Russia

e-mail: r_n_karasev@mail.ru

R. Karasev

Laboratory of Discrete and Computational Geometry, Yaroslavl State University, Sovetskaya st. 14, Yaroslavl 150000, Russia 
There are results about lowering the Carathéodory constant: A theorem of Fenchel [11] (see also [12]) asserts that a compactum $X \subset \mathbb{R}^{n}$ either has the Carathéodory number $\leq n$ or can be separated by a hyperplane into two nonempty parts. By separated we mean "divided by a hyperplane disjoint from $X$ into two nonempty parts.” In order to state more results, we need formal definitions.

Definition 1.1 For a compactum $X \subset \mathbb{R}^{n}$, we denote by $\operatorname{conv}_{k} X$ the set of points $p \in \mathbb{R}^{n}$ that can be expressed as a convex combination of at most $k$ points in $X$. We denote by conv $X$ (without subscript) the standard convex hull of $X$.

Definition 1.2 The Carathéodory number of $X$ is the smallest $k$ such that conv $X=$ $\operatorname{conv}_{k} X$.

Remark 1.3 So, when $X \subset \mathbb{R}^{n}$, Carathéodory's theorem [7] is equivalent to the equality conv $X=\operatorname{conv}_{n+1} X$. We will give an alternative definition for $\operatorname{conv}_{k} X$ in Sect. 4 as the $k$-fold join of $X$.

Definition 1.4 A compactum $X \subset \mathbb{R}^{n}$ is $k$-convex if every linear image of $X$ to $\mathbb{R}^{k}$ is convex.

We give some examples of $k$-convex sets. What is needed in Fenchel's theorem is 1 -convexity, and every connected set is 1 -convex. The $k$-skeleton of a convex polytope is $k$-convex (though for such $k$-convex sets, most results of this paper are trivial). In [6] (see also [5, Chap. II, Sect. 14]) it is shown that the image of the sphere under the Veronese map $v_{2}: S^{n-1} \rightarrow \mathbb{R}^{n(n+1) / 2}$ (with all degree 2 monomials as coordinates) is 2-convex.

In [12, Corollary 1] the following remarkable result is proved:

Theorem 1.5 (Hanner-Rådström, 1951) If $X$ is a union of at most $n$ compacta $X_{1}, \ldots, X_{n}$ in $\mathbb{R}^{n}$ and each $X_{i}$ is 1-convex, then $\operatorname{conv}_{n} X=\operatorname{conv} X$.

It is also known $[5,15]$ that a convex curve in $\mathbb{R}^{n}$ (that is, a curve with no $n+1$ points in a single affine hyperplane) has Carathéodory number at most $\left\lfloor\frac{n+2}{2}\right\rfloor$. It would be interesting to obtain some nontrivial bounds for the Carathéodory number of the orbit $G x$ of a point $x$ in a representation $V$ of a compact Lie group $G$ in terms of $\operatorname{dim} V$ and $\operatorname{dim} G$ (or the rank of $G$ ). The latter question is mentioned in [18, Question 3] and would be useful in results like those in [17].

In Sects. 2 and 3 of this paper we show that the Carathéodory number is at most $k+1$ for $(n-k)$-convex sets. In Sect. 4 we prove the corresponding analogue of the colorful Carathéodory theorem, and in Sect. 5 we develop another topological approach to colorful Carathéodory-type results, which may be of independent interest. In Sect. 6 we give a related Tverberg-type result.

We note that the content of Sects. 2-4 may be trivially generalized to positive hulls in place of convex hulls, as was done, for example, in [2]. But we do not state the corresponding results explicitly to simplify the exposition. 


\section{The Carathéodory Number and $k$-Convexity}

We are going to give a natural generalization of the reasoning in [12]:

Theorem 2.1 Suppose that $X_{1}, \ldots, X_{n-k}$ are compacta in $\mathbb{R}^{n}$ and $p$ does not belong to $\operatorname{conv}_{k+1} X_{i}$ for any $i$. Then there exists an affine $k$-plane $L \ni p$ that has empty intersection with any $X_{i}$.

Remark 2.2 If we replace $\operatorname{conv}_{k+1} X_{i}$ by the honest convex hull conv $X_{i}$, then the result is simply deduced by induction from the Hahn-Banach theorem.

Remark 2.3 In [16] a somewhat related result was proved: For a compactum $X \subset \mathbb{R}^{n}$ and a point $p \notin X$, there exists an affine $k$-plane $L$ (for a prescribed $k<n$ ) such that the intersection $L \cap K$ is not acyclic modulo 2. Here acyclic means having the Čech cohomology of a point.

The proof of Theorem 2.1 is given in Sect. 3. Now we deduce the following generalization of Fenchel's theorem [11] (stated in the second paragraph of the introduction):

Corollary 2.4 If a compactum $X \subset \mathbb{R}^{n}$ is $(n-k)$-convex, then $\operatorname{conv}_{k+1} X=\operatorname{conv} X$.

Proof Assume the contrary and let $p \in \operatorname{conv} X \backslash \operatorname{conv}_{k+1} X$. Applying Theorem 2.1 to the family $\underbrace{X, \ldots, X}_{n-k}$, we find a $k$-dimensional $L \ni p$ disjoint from $X$. Now project $X$ along $L$ with $\pi: \mathbb{R}^{n} \rightarrow \mathbb{R}^{n-k}$. Since $X$ is $(n-k)$-convex, $\pi(L)$ must be separated from $\pi(X)$ by a hyperplane. Hence, $L$ is separated from $X$ by a hyperplane, and therefore $p$ cannot be in conv $X$.

Remark 2.5 In the above Corollary 2.4 and its proof we could consider $n-k$ different $(n-k)$-convex compacta $X_{1}, \ldots, X_{n-k}$ and by the same reasoning obtain the following conclusion:

$$
\bigcup_{i=1}^{n-k} \operatorname{conv}_{k+1} X_{i}=\bigcup_{i=1}^{n-k} \operatorname{conv} X_{i} .
$$

But this result trivially follows from Corollary 2.4 by taking the union.

Remark 2.6 For the image $v_{2}\left(S^{n-1}\right)$ of the Veronese map, the Carathéodory constant is roughly of order $n$, see [5, Chap. II, Sect. 14, Theorem 14.3]. Hence, Corollary 2.4 is not optimal for this set.

\section{Proof of Theorem 2.1}

Let us replace $X_{i}$ by a smooth nonnegative function $\rho_{i}$ such that $\rho_{i}>0$ on $X_{i}$ and $\rho_{i}=0$ outside some $\varepsilon$-neighborhood of $X_{i}$. Let $p$ be the origin. 
Assume the contrary: For any $k$-dimensional linear subspace $L \subset \mathbb{R}^{n}$ some intersection $L \cap X_{i}$ is nonempty. The space of all possible $L$ is the Grassmann manifold $G_{n}^{k}$. Denote by $D_{i}$ the open subset of $G_{n}^{k}$ consisting of $L \in G_{n}^{k}$ such that $\int_{L} \rho_{i}>0$. Note that 0 cannot lie in the convex hull conv $\left(L \cap X_{i}\right)$ because in this case by the ordinary Carathéodory theorem, 0 would be in $\operatorname{conv}_{k+1}\left(L \cap X_{i}\right) \subseteq \operatorname{conv}_{k+1} X_{i}$, contradicting the hypothesis. Hence (if we choose small enough $\varepsilon>0$ ), the "momentum" integral

$$
m_{i}(L)=\int_{L} \rho_{i} x d x
$$

never coincides with 0 over $D_{i}$. Obviously, $m_{i}(L)$ is a continuous section of the canonical vector bundle $\gamma: E(\gamma) \rightarrow G_{n}^{k}$, which is nonzero over $D_{i}$. Now we apply the following:

Lemma 3.1 Any $n-k$ sections of $\gamma: E(\gamma) \rightarrow G_{n}^{k}$ have a common zero because of the nonzero Euler class $e(\gamma)^{n-k}$.

This lemma is a folklore fact, see, for example, [9, 22]. Applying this lemma to the sections $m_{i}$, we obtain that the sets $D_{i}$ do not cover the entire $G_{n}^{k}$. Hence, some $L \in G_{n}^{k}$ has an empty intersection with every $X_{i}$.

Remark 3.2 In the proof of Theorem 1.5 in [12, the proof of Theorem 3] Hanner and Rådström find a maximum of certain volume function over a convex subset of the sphere $S^{n-1}$. This may also be done by an application of the Brouwer fixed-point theorem similar to the above proof, thus exhibiting the topological nature of that result.

\section{The Colorful Carathéodory Number}

Let us introduce some notation and restate the colorful Carathéodory theorem [2].

Definition 4.1 Denote by $A * B$ the geometric join of two sets $A, B \subseteq \mathbb{R}^{n}$, which is

$$
\{t a+(1-t) b: a \in A, b \in B \text {, and } t \in[0,1]\} \text {. }
$$

This is actually the alternative definition of $\operatorname{conv}_{k} X$ as $\underbrace{X * \cdots * X}_{k}$.

Theorem 4.2 (Bárány, 1982) If $X_{1}, \ldots, X_{n+1} \subset \mathbb{R}^{n}$ are compacta and $0 \in \operatorname{conv} X_{i}$ for every $i$, then $0 \in X_{1} * X_{2} * \cdots * X_{n+1}$.

It is possible to reduce the Carathéodory number $n+1$ assuming the $(n-k)$ convexity of $X_{i}$, thus generalizing Corollary 2.4:

Theorem 4.3 Let $0 \leq k \leq n$. If $X_{1}, \ldots, X_{k+1} \subset \mathbb{R}^{n}$ are $(n-k)$-convex compacta and $0 \in \operatorname{conv} X_{i}$ for every $i$, then $0 \in X_{1} * X_{2} * \cdots * X_{k+1}$. 
Proof We use the classical scheme [2] along with the degree reasoning used in [1, 4, $8,19]$ in the proof of different generalizations of the colorful Carathéodory theorem.

First consider the case $k=n-1$. In this case we have $n$ sets and 1-convexity. Let $x_{1}, \ldots, x_{n}$ be the system of representatives of $X_{1}, \ldots, X_{n}$ such that the distance from $S=\operatorname{conv}\left\{x_{1}, \ldots, x_{n}\right\}$ to the origin is minimal. If this distance is zero, then we are done. Otherwise, assume that $z \in S$ minimizes the distance.

Let $z=t_{1} x_{1}+\cdots+t_{n} x_{n}$, a convex combination of the $x_{i} \mathrm{~s}$. If $t_{i}=0$, then we observe that $0 \in \operatorname{conv} X_{i}$, and we can replace $x_{i}$ by another $x_{i}^{\prime}$ so that new simplex $S^{\prime}=\operatorname{conv}\left\{x_{1}, \ldots, x_{i-1}, x_{i}^{\prime}, x_{i+1}, \ldots, x_{n}\right\}$ is closer to the origin than $S$. So we may assume that all the coefficients $t_{i}$ are positive and $z$ is in the relative interior of $S$. This also implies that $S$ is $(n-1)$-dimensional, i.e., there is a unique hyperplane containing $S$.

Consider the hyperplane $h \ni 0$ parallel to $S$. Applying the definition of 1-convexity to the projection along $h$, we obtain that there exists a system of representatives $y_{i} \in$ $X_{i} \cap h$. The set

$$
f(B)=\left\{x_{1}, y_{1}\right\} *\left\{x_{2}, y_{2}\right\} * \cdots *\left\{x_{n}, y_{n}\right\}
$$

is a piecewise linear image of the boundary of a crosspolytope, which we denote by $B$. Note that for every facet $F$ of $B$, the vertices of the simplex $f(F)$ form a system of representatives for $\left\{X_{1}, \ldots, X_{n}\right\}$. In particular, $S=f(F)$ for some facet $F$ of $B$. The line $\ell$ through the origin and $z$ intersects the simplex $S=f(F)$ transversally, and so it must intersect some other $f\left(F^{\prime}\right)$ (where $F^{\prime} \neq F$ is a facet of $B$ ) because of the parity of the intersection index. The intersection $\ell \cap f\left(F^{\prime}\right)$ is on the segment $[0, z]$ and cannot coincide with $z$. Therefore, $f\left(F^{\prime}\right)$ is closer to the origin than $S$. This is a contradiction with the choice of $S$. Thus, the case $k=n-1$ is done.

The case $k=0$ of this theorem is trivial by definition, and the case $k=n$ corresponds to the colorful Carathéodory theorem. Now let $0<k<n-1$. Consider again a system of representatives $x_{1}, \ldots, x_{k+1}$ minimizing the distance $\operatorname{dist}\left(0, \operatorname{conv}\left\{x_{1}, \ldots, x_{k+1}\right\}\right)$. Put $S=\operatorname{conv}\left\{x_{1}, \ldots, x_{k+1}\right\}$. As above, the closest to the origin point $z \in S$ must lie in the relative interior of $S$ if $z \neq 0$.

Let $L \subset \mathbb{R}^{n}$ be the $k$-dimensional linear subspace parallel to $S$. As in the first proof, using $(n-k)$-convexity, we select $y_{i} \in L \cap X_{i}$. Then we map naturally the boundary $B$ of a $(k+1)$-dimensional crosspolytope to the geometric join

$$
f(B)=\left\{x_{1}, y_{1}\right\} *\left\{x_{2}, y_{2}\right\} * \cdots *\left\{x_{k+1}, y_{k+1}\right\} .
$$

Note that $f(B)$ is contained in the $(k+1)$-dimensional linear span of $S$ and $L$, so by the parity argument as above the image under $f$ of some face of $B$ must be closer to the origin than $S$.

Remark 4.4 In this proof in the case $k<n-1$ we can choose some $(k+1)$ dimensional subspace $M \subset \mathbb{R}^{n}$ and a system of representatives $\left\{x_{1}, \ldots, x_{k+1}\right\}$ for $M \cap X_{1}, \ldots, M \cap X_{k+1}$. Then we can make the steps reducing $\operatorname{dist}\left(0, \operatorname{conv}\left\{x_{1}, \ldots\right.\right.$, $\left.x_{k+1}\right\}$ ) so that the system of representatives always remains in $M$. 


\section{A Topological Approach to Theorem 4.3}

Theorem 4.3 can also be deduced from the following lemma:

Lemma 5.1 Let $\xi: E(\xi) \rightarrow X$ be a $k$-dimensional vector bundle over a compact metric space $X$. Let $Y_{1}, \ldots, Y_{k+1}$ be closed subspaces of $E(\xi)$ such that for every $i$, the projection $\left.\xi\right|_{Y_{i}}: Y_{i} \rightarrow X$ is surjective. If $e(\xi) \neq 0$, then for some fiber $V=\xi^{-1}(x)$, the geometric join

$$
\left(Y_{1} \cap V\right) * \cdots *\left(Y_{k+1} \cap V\right)
$$

contains $0 \in V$.

Remark 5.2 The Euler class here may be considered in integral cohomology or in the cohomology mod 2. The proof passes in both cases, so we omit the coefficients from the notation.

Reduction of Theorem 4.3 to Lemma 5.1 for $k<n$ Take a linear subspace $M \subseteq \mathbb{R}^{n}$ of dimension $k+1$. For every $k$-dimensional linear subspace $L \subset M$, all the intersections $L \cap X_{i}$ are nonempty. All such $L$ constitute the canonical bundle $\gamma$ over $G_{k+1}^{k}=\mathbb{R} P^{k}$ with nonzero Euler class by Lemma 3.1. For any fixed $i$, the union of sets $L \cap X_{i}$ constitutes a closed subset of $E(\gamma)$ that we denote by $Y_{i}$. By Lemma 5.1, for some $L$, the join

$$
\left(Y_{1} \cap L\right) * \cdots *\left(Y_{k+1} \cap L\right)=\left(X_{1} \cap L\right) * \cdots *\left(X_{k+1} \cap L\right)
$$

must contain the origin.

Now we prove Lemma 5.1 The proof has much in common with the results of [16]. The main idea is that fiberwise acyclic (up to some dimension) subsets of the total space of a vector bundle behave like sections of that vector bundle.

Let $Y=Y_{1} *_{X} * \cdots *_{X} Y_{k+1}$ be the abstract fiberwise join over $X$, that is, the set of all formal convex combinations

$$
t_{1} y_{1}+t_{2} y_{2}+\cdots+t_{k+1} y_{k+1}
$$

where $t_{i}$ are nonnegative reals with unit sum, and $y_{i} \in Y_{i}$ are points such that

$$
\xi\left(y_{1}\right)=\cdots=\xi\left(y_{k+1}\right) .
$$

Denote the natural projection $\eta: Y \rightarrow X$. Any formal convex combination $y \in Y$ defines a corresponding "geometric" convex combination $f(y)$ in the fiber $\xi^{-1}(\eta(y))$ depending continuously on $y$. It is easy to check that $f(y)$ can be considered as a section of the pullback vector bundle $\eta^{*}(\xi)$ over $Y$.

For any point $x \in X$, its preimage under $\eta$ is a join of $(k+1)$ nonempty sets

$$
\left(Y_{1} \cap \xi^{-1}(x)\right) * \cdots *\left(Y_{k+1} \cap \xi^{-1}(x)\right),
$$


and therefore $\eta^{-1}(x)$ is $(k-1)$-connected. Hence, the Leray spectral sequence for the Čech cohomology $H^{*}(Y)$ with $E_{2}^{*, *}=H^{*}\left(X ; \mathcal{H}^{*}\left(\eta^{-1}(x)\right)\right)$ (the coefficient sheaf is the direct image of the homology of the total space) has empty rows number $1, \ldots, k-1$, and its differentials cannot kill the image of $e(\xi)$ in $E_{r}^{k, 0}$. Hence, $\eta^{*}(e(\xi))=e\left(\eta^{*}(\xi)\right)$ remains nonzero over $Y$, and by the standard property of the Euler class, for some $y \in Y$, the section $f(y)$ must be zero.

Remark 5.3 In this proof we essentially use the inequality $k<n$. So the colorful Carathéodory theorem is not a consequence of Lemma 5.1, at least in our present state of knowledge.

The subsets $Y_{i}$ in Lemma 5.1 can be considered as set-valued sections. The same technique proves the following:

Theorem 5.4 Let $B$ be an $n$-dimensional ball, and $f_{i}: B \rightarrow 2^{B} \backslash \emptyset$ for $i=1, \ldots$, $n+1$ be set-valued maps with closed graphs (in $B \times B$ ). Then for some $x \in B$, we have the inclusion

$$
x \in f_{1}(x) * \cdots * f_{n+1}(x) .
$$

Proof We may assume that all sets $f_{i}(x)$ are in the interior of $B$, because the general case is reduced to this one by composing $f_{i}$ with a homothety with scale $1-\varepsilon$ and going to the limit as $\varepsilon \rightarrow+0$.

It is known [14] that for a single-valued map $f: B \rightarrow$ int $B$ (considered as a section of the trivial bundle $\left.B \times \mathbb{R}^{n} \rightarrow B\right)$, a fixed point $(x=f(x))$ is guaranteed by the relative Euler class $e(f(x)-x) \in H^{n}(B, \partial B)$. Then the proof proceeds as in Lemma 5.1 by lifting $e(f(x)-x)$ to the abstract fiberwise join of graphs of $f_{i}$ over the pair $(B, \partial B)$ and using the properties of the relative Euler class of a section.

Corollary 5.5 Suppose that $X_{1}, \ldots, X_{n+1}$ are compacta in $\mathbb{R}^{n}$ and $\rho$ is a continuous metric on $\mathbb{R}^{n}$. For any $x \in \mathbb{R}^{n}$, denote by $f_{i}(x)$ the set of farthest from $x$ points in $X_{i}$ (in the metric $\rho$ ). Then, for some $x \in \mathbb{R}^{n}$, we have

$$
x \in f_{1}(x) * \cdots * f_{n+1}(x) .
$$

Remark 5.6 If we denote by $f_{i}(x)$ the closets points in $X_{i}$, then this assertion becomes almost trivial without using any topology.

\section{The Carathéodory Number and the Tverberg Property}

Tverberg's classical theorem [20] is the following:

Theorem 6.1 (Tverberg, 1966) Every set of $(n+1)(r-1)+1$ points in $\mathbb{R}^{n}$ can be partitioned into $r$ parts $X_{1}, \ldots, X_{r}$ so that the convex hulls conv $X_{i}$ have a common point. 
From the general position considerations it is clear that the number $(n+1) \times$ $(r-1)+1$ cannot be decreased. But we are going to decrease it after replacing a finite point set by a family of convex compacta. Let us define the Carathéodory number for such families.

Definition 6.2 Let $\mathcal{F}$ be a family of convex compacta in $\mathbb{R}^{n}$. The Carathéodory number of $\mathcal{F}$ is the least $\kappa$ such that for any subfamily $\mathcal{G} \subseteq \mathcal{F}$,

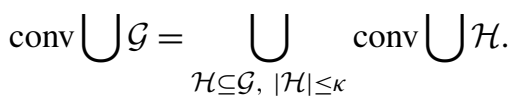

We denote the Carathéodory number of $\mathcal{F}$ by $\kappa(\mathcal{F})$.

Again, from the Carathéodory theorem [7] it follows that $\kappa(\mathcal{F}) \leq n+1$. Another observation is that Corollary 2.4 guarantees that $\kappa(\mathcal{F}) \leq k+1$ if the union of every subfamily $\mathcal{G} \subseteq \mathcal{F}$ is $(n-k)$-convex.

Now we state the analogue of Tverberg's theorem.

Theorem 6.3 Suppose that $\mathcal{F}$ is a family of convex compacta in $\mathbb{R}^{n}, r$ is a positive integer, and

$$
|\mathcal{F}| \geq \operatorname{r\kappa }(\mathcal{F})+1 \text {. }
$$

Then $\mathcal{F}$ can be partitioned into $r$ subfamilies $\mathcal{F}_{1}, \ldots, \mathcal{F}_{r}$ so that

$$
\bigcap_{i=1}^{r} \operatorname{conv} \bigcup \mathcal{F}_{i} \neq \emptyset \text {. }
$$

Remark 6.4 Note the following: If $\kappa(\mathcal{F})=n+1$, then taking a system of representatives for $\mathcal{F}$ and applying the Tverberg theorem, we obtain a weaker condition: $|\mathcal{F}| \geq(r-1)(n+1)+1$.

Remark 6.5 This theorem originated in discussions with Andreas Holmsen, who established the same result in the special case $n=2, \kappa(\mathcal{F})=2$, and with $|\mathcal{F}| \geq 2 r$ (not $2 r+1)$, see [13]. Together with the previous remark, this shows that the condition on $|\mathcal{F}|$ may be not tight, though we have no idea how to improve it in the general case.

Proof of Theorem 6.3 We again use a minimization argument, combined with Sarkaria's trick [21] in the more convenient tensor form, which is from [3].

Let $|\mathcal{F}|=m, \kappa=\kappa(\mathcal{F})$, and

$$
\mathcal{F}=\left\{C_{1}, C_{2}, \ldots, C_{m}\right\} .
$$

Put the space $\mathbb{R}^{n}$ to $A=\mathbb{R}^{n+1}$ as a hyperplane given by the equation $x_{n+1}=1$. Consider a set $S$ of vertices of a regular $(r-1)$-simplex in some $(r-1)$-dimensional space $V$ and assume that $S$ is centered at the origin.

Now define the subsets of $V \otimes A$ by

$$
X_{i}=S \otimes C_{i}
$$


and consider a system of representatives $\left(x_{1}, x_{2}, \ldots, x_{m}\right)$ for the family of sets $\mathcal{G}=$ $\left\{X_{1}, X_{2}, \ldots, X_{m}\right\}$. Such a system gives rise to a partition $\left\{P_{s}: s \in S\right\}$ of $\{1, \ldots, m\}$ in the following way. For $s \in S$, define

$$
P_{s}=\left\{i \in\{1, \ldots, m\}: x_{i}=s \otimes c_{i} \text { for some } c_{i} \in C_{i}\right\}
$$

Like in [1, Lemma 2], we observe that $0 \in \operatorname{conv}\left\{x_{1}, \ldots, x_{m}\right\}$ if and only if $\bigcap_{s \in S} \operatorname{conv}\left\{c_{i}: i \in P_{s}\right\} \neq \emptyset$. Based on this, we choose a system of representatives $\left(x_{1}, \ldots, x_{m}\right)$ of $\mathcal{G}$ so that the distance between 0 and $\operatorname{conv}\left\{x_{1}, x_{2}, \ldots, x_{m}\right\}$ is minimal. If this distance is zero, then the required partition of $\mathcal{F}$ is given by the sets $\left\{C_{i} \in \mathcal{F}: i \in P_{S}\right\}, s \in S$.

Assume that the minimal distance is not zero. Then it is attained on some convex combination

$$
x_{0}=\alpha_{1} x_{1}+\alpha_{2} x_{2}+\cdots+\alpha_{m} x_{m} .
$$

We claim that $\alpha_{i}>0$ for all $i \in\{1, \ldots, m\}$. Assume, for instance, that $\alpha_{1}=0$ and $x_{1}=s \otimes c_{1}$ for some $c_{1} \in C_{1}$ and $s \in S$. Now $x_{1}$ can be replaced by $t \otimes c_{1}$ for any $t \in S$ as such a change does not influence $x_{0}$. The distance minimality condition implies that all the points $t \otimes c_{1}$ are separated from the origin by a hyperplane in $V \otimes A$, which is the support hyperplane for the ball, centered at the origin and touching conv $\left\{x_{1}, \ldots, x_{m}\right\}$. Obviously,

$$
\sum_{t \in S} t \otimes c_{i}=0
$$

so the points $t \otimes c_{i}, t \in S$, are not separated from the origin. This contradiction completes the proof of the claim.

The above convex combination representing $x_{0}$ can be written as

$$
x_{0}=\sum_{s \in S} s \otimes\left(\sum_{i \in P_{s}} \alpha_{i} c_{i}\right) \text {. }
$$

Assume first that none of $P_{s}$ is the empty set. Define $c(s)=\sum_{i \in P_{s}} \alpha_{i} c_{i}$ and $\alpha(s)=$ $\sum_{i \in P_{s}} \alpha_{i}>0$. Then $c(s) / \alpha(s)$ is a convex combination of elements $c_{i} \in C_{i}, i \in P_{s}$. Thus, $c(s) / \alpha(s) \in \operatorname{conv} \bigcup_{i \in P_{s}} C_{i}$. According to the definition of the Carathéodory number, there is a subset $P_{s}^{\prime} \subset P_{s}$, of size at most $\kappa$, such that $c(s) / \alpha(s) \in$ conv $\bigcup_{i \in P_{s}^{\prime}} C_{i}$ for every $s \in S$. This means that there are $c_{i}^{\prime} \in C_{i}$ for all $i \in P_{s}^{\prime}$ such that $c(s) / \alpha(s) \in \operatorname{conv}\left\{c_{i}^{\prime}: i \in P_{s}^{\prime}\right\}$; in other words, $c(s)=\sum_{i \in P_{s}^{\prime}} \alpha_{i}^{\prime} c_{i}^{\prime}$ with positive $\alpha_{i}^{\prime}$ satisfying $\sum_{i \in P_{s}^{\prime}} \alpha_{i}^{\prime}=\alpha(s)$. Thus,

$$
x_{0}=\sum_{s \in S} s \otimes\left(\sum_{i \in P_{s}^{\prime}} \alpha_{i}^{\prime} c_{i}\right) .
$$

In this case the minimum distance is attained on the convex hull of no more that $r \kappa$ elements as each $\left|P_{s}^{\prime}\right| \leq \kappa$. But $m>r \kappa$, contradicting the claim. 
Finally, we have to deal with the (easy) case where some $P_{s}=\emptyset$. The above argument works, with no change at all, for the nonempty $P_{s}$, implying that $x_{0}$ can be written as a convex combination of at most $(r-1) \kappa$ elements. Again, $m>(r-1) \kappa$, and the same contradiction finishes the proof.

Acknowledgements We thank Peter Landweber who has drawn our attention to those old results by Fenchel, Hanner, and Rådström and Alexander Barvinok for discussions and examples of $k$-convexity.

The work of both authors was supported by ERC Advanced Research Grant No 267195 (DISCONV). The first author acknowledges support from Hungarian National Research Grant No 78439. The second author is supported by the Dynasty Foundation, the President's of Russian Federation grant MD-352.2012.1, the Russian Foundation for Basic Research grants 10-01-00096 and 10-01-00139, the Federal Program "Scientific and scientific-pedagogical staff of innovative Russia" 2009-2013, and the Russian government project 11.G34.31.0053.

\section{References}

1. Arocha, J., Bárány, I., Bracho, J., Fabila, R., Montejano, L.: Very colorful theorems. Discrete Comput. Geom. 42(2), 142-154 (2009)

2. Bárány, I.: A generalization of Carathéodory's theorem. Discrete Math. 40, 141-152 (1982)

3. Bárány, I., Onn, S.: Colourful linear programming and its relatives. Math. Oper. Res. 22, 550-567 (1997)

4. Bárány, I., Matoušek, J.: Quadratically many colorful simplices. SIAM J. Discrete Math. 21, 191-198 (2007)

5. Barvinok, A.: A Course in Convexity. Graduate Studies in Mathematics, vol. 54. AMS, Providence (2002)

6. Brickman, L.: On the field of values of a matrix. Proc. Am. Math. Soc. 12(1), 61-66 (1961)

7. Carathéodory, C.: Über den Variabilitätsbereich der Fourierschen Konstanten von positiven harmonischen Funktionen. Rend. Circ. Mat. Palermo 32, 193-217 (1911)

8. Deza, A., Huang, S., Stephen, T., Terlaky, T.: Colourful simplicial depth. Discrete Comput. Geom. 35(4), 597-615 (2006)

9. Dol'nikov, V.L.: Transversals of families of sets in $\mathbb{R}^{n}$ and a connection between the Helly and Borsuk theorems. Russ. Acad. Sci. Sb. Math. 79(1), 93-107 (1994). 777-780 (1987)

10. Eckhoff, J.: Helly, Radon, and Carathéodory type theorems. In: Gruber, P.M., Willis, J.M. (eds.) Handbook of Convex Geometry, pp. 389-448. North-Holland, Amsterdam (1993)

11. Fenchel, W.: Über Krümmung und Windung geschlossener Raumkurven. Math. Ann. 101, 238-252 (1929)

12. Hanner, O., Rådström, H.: A generalization of a theorem of Fenchel. Proc. Am. Math. Soc. 2, 589-593 (1951)

13. Holmsen, A.: Geometric transversal theory: T(3)-families in the plane. In: Geometry-Intuitive, Discrete, and Convex: A Tribute to László Fejes Tóth, ed. by I. Bárány, K.J. Böröczky, G. Fejes Tóth, J. Pach, Bolyai Society Mathematical Studies, Vol. 24. Springer, Berlin (2012, to appear)

14. Karasev, R.N.: Dual theorems on central points and their generalizations. Russian Acad. Sci. Sb. Math. 199(10), 1459-1479 (2008). A corrected version is available at arXiv:0909.4915

15. Karlin, S., Studden, W.J.: Tchebycheff Systems: With Applications in Analysis and Statistics. Interscience Publishers, New York (1966)

16. Kosiński, A.: A theorem on families of acyclic sets and its applications. Pac. J. Math. 12(1), 317-325 (1962)

17. Milman, V.D.: A few observations on the connections between local theory and some other fields. In: Geometric Aspects of Functional Analysis. Lecture Notes in Mathematics, vol. 1317, pp. 283-289 (1988)

18. Sanyal, R., Sottile, F., Sturmfels, B.: Orbitopes (2009). arXiv:0911.5436

19. Stephen, T., Thomas, H.: A quadratic lower bound for colourful simplicial depth. J. Comb. Optim. 16(4), 324-327 (2008)

20. Tverberg, H.: A generalization of Radon's theorem. J. Lond. Math. Soc. 41, 123-128 (1966)

21. Sarkaria, K.S.: Tverberg's theorem via number fields. Isr. J. Math. 79, 317-320 (1992)

22. Živaljević, R.T., Vrećica, S.T.: An extension of the ham sandwich theorem. Bull. Lond. Math. Soc. 22(2), 183-186 (1990) 